\title{
Analysis of the role of hole transport layer materials to the performance of perovskite solar cell
}

\author{
Istighfari Dzikri ${ }^{1}$, Michael Hariadi ${ }^{1}$, Retno Wigajatri Purnamaningsih ${ }^{1}$, and Nji Raden Poespawati ${ }^{1, *}$ \\ ${ }^{1}$ Department of Electrical Engineering, Faculty of Engineering, Universitas Indonesia, Depok 16424, Indonesia
}

\begin{abstract}
Research in solar cells is needed to maximize Indonesia's vast solar potential that can reach up to $207.898 \mathrm{MW}$ with an average radiation of $4.8 \mathrm{kWh} / \mathrm{m}^{2} /$ day. Organometallic perovskite solar cells (PSCs) have gained immense attention due to their rapid increase in efficiency and compatibility with low-cost fabrication methods. Understanding the role of hole transport layer is very important to obtain highly efficient PSCs. In this work, we studied the effect of Hole Transport Layer (HTL) to the performance of perovskite solar cell. The devices with HTL exhibit substantial increase in power conversion efficiency, open circuit voltage and short circuit current compared to the device without HTL. The best performing device is PSC with CuSCN as HTL layer, namely Voc of 0.24 , Isc of $1.79 \mathrm{~mA}, 0.27 \mathrm{FF}$ and efficiency of $0.09 \%$.
\end{abstract}

\section{Introduction}

Indonesia is a tropical country with high solar potential that can reach up to 207,898 MW with an average radiation of $4.8 \mathrm{kWh} / \mathrm{m} 2 /$ day $^{[1-2]}$. The application of photovoltaic devices is one of the most promising solutions for satisfying Indonesia's energy demand. Organometallic perovskite solar cells have gained immense attention due to their compatibility with lowcost fabrication methods and high power conversion efficiency, certified up to $22.7 \%$ by the National Renewable Energy Laboratory ${ }^{[3]}$. Organometallic perovskite, such as $\mathrm{CH}_{3} \mathrm{NH}_{3} \mathrm{PbX}_{3}(\mathrm{X}=\mathrm{I}, \mathrm{Br}, \mathrm{Cl})$, has the characteristics of low cost fabrication technique with roll-to-roll or solution processing, high absorption coefficient and long diffusion length, making them one of the most promising photovoltaic material for cost effective solar cell ${ }^{[4-6]}$.

PEDOT:PSS is a hybrid conducting polymer system that is widely used in organic optoelectronics due to its tuneable conductivity and high optical transmittance in the visible light wavelength range ${ }^{[7]}$. However, the commonly used PEDOT:PSS solution is acidic and may react with the perovskite due to the existence of the basic methyl amine in the structure of organometal trihalide perovskites. PEDOT:PSS is also often coated from its aqueous solution. The PSS content in the PEDOT:PSS hybrid system is hygroscopic and may induce the potential instability since the organometal trihalide perovskite is unstable under humid environments ${ }^{[8-9]}$. These two reasons make processing of PEDOT:PSS directly on top of the perovskite film challenging. Here, we made the PEDOT:PSS layer on another FTO before sandwiching them together to minimize the risk of degradation from the perovskite layer, especially under high humidity condition (50$60 \%$ ) in which the fabrication is done. Copper thiocyanate $(\mathrm{CuSCN})$ has also been recently applied as hole transporting materials for perovskite solar cells. This inorganic p-type semiconductor shows good transparency, high hole mobility of $0.01-0.1 \mathrm{~cm}^{2} \mathrm{~V}^{-1} \mathrm{~s}^{-1}$ (as compared with $4 \times 10^{-5} \mathrm{~cm}^{2} \mathrm{~V}^{-1} \mathrm{~S}^{-1}$ for spiroOMeTAD) and good chemical stability ${ }^{[10]}$. Some researchers have tried to explain the role of hole transport layer (HTL), however the research focused on the specific role of spiro-OMeTAD instead of the general advantage of using HTL ${ }^{[11]}$ or focused on the role of HTL in an inverted (p-i-n) configuration ${ }^{[12]}$ instead of the normal n-i-p configuration used in this paper. In this paper, we present the detailed device fabrication methods for two different hole transporting materials, (CuSCN and PEDOT:PSS) and compare the different device performance of the cells with and without HTL to analyse the role of HTL and explore the effect of using different materials to the performance of perovskite solar cell.

\section{Methodology}

\subsection{Structure}

The fabricated perovskite solar cell with $\mathrm{CuSCN}$ is similar to the configuration as reported by Sameer Patwardhan et $\mathrm{a}^{[13]}$, pictured in Figure 1a. In Figure 1b,

\footnotetext{
* Corresponding author: pupu@eng.ui.ac.id
} 
we illustrated the structure of the PSC with PEDOT:PSS. The cell without HTL is similar to the one pictured in Figure 1a but without the CuSCN layer.

\begin{tabular}{c} 
Glass \\
FTO \\
\hline Carbon Powder \\
CuSCN \\
Perovskite \\
TiO2 \\
FTO \\
Glass \\
(a) \\
Glass \\
FTO \\
PEDOT:PSS \\
Carbon powder \\
Perovskite \\
TiO2 \\
FTO \\
Glass
\end{tabular}

(b)

Fig. 1. Structure of the fabricated solar cells for (a) CuSCN and (b) PEDOT:PSS

In this paper, $\mathrm{TiO}_{2}$ and $\mathrm{CuSCN}$ is used as an electron transport layer and hole transport layer, respectively because they do not absorb visible light due to their wide band gap and exhibit fast electron and hole transport. Interfacial layers, in particular the electron transport layer, should not absorb visible light to maximize the amount of light absorbed by the perovskite layer. CuSCN has higher hole mobility than the normally used spiro-OMeTAD and has good chemical stability, making them a good alternative to the expensive spiro-OMeTAD ${ }^{[10]}$. PEDOT:PSS is used because of their high optical transmittance in the visible light wavelength range, their wide availability in the general market and their ability to be deposited on flexible material for future commercial use ${ }^{[7]}$. Figure 2 shows the energy level $(\mathrm{eV})$ of the fabricated cells. FTO glass used in this paper has an energy level of $4.4 \mathrm{eV}$ according to the research by Quinten A.et al ${ }^{[14]}$. Energy levels of $\mathrm{TiO}_{2}$, perovskite and carbon was taken from the research conducted by Heng Wang et al ${ }^{[15]}$. CuSCN's energy level was taken directly from Peng Qin's works ${ }^{[16]}$ while PEDOT:PSS's energy level varies from -4.9 to $-5.2 \mathrm{eV}$ but the PEDOT:PSS used here is the same as the research by Hobeom Kim et al, having an energy level of around $-4.9 \mathrm{eV}^{[12]}$.

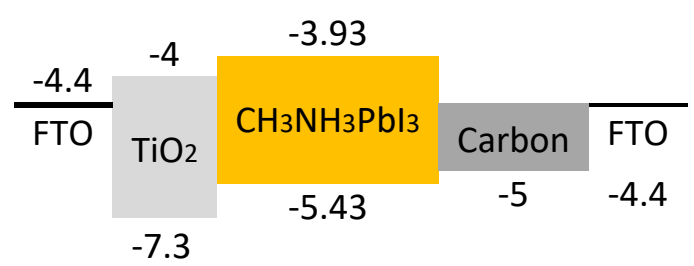

(a)

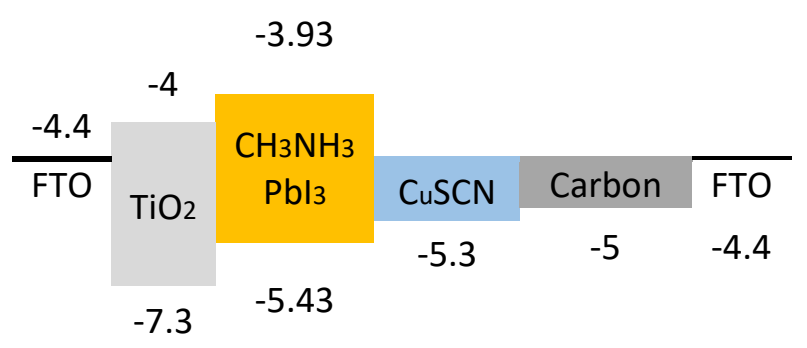

(b)

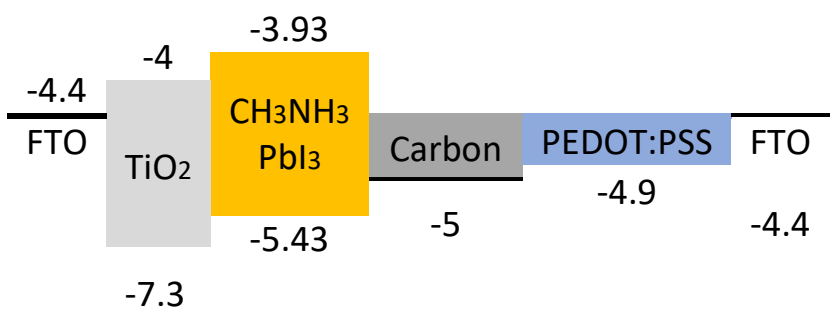

(c)

Fig. 2. Energy level of the fabricated solar cells for (a) cell without HTL, (b) CuSCN and (c) PEDOT:PSS

The perovskite layer, with a band gap of $\sim 1.5 \mathrm{eV}$, absorbs light and creates electrons in the Conduction Band (CB) and holes in the Valence Band (VB). $\mathrm{TiO}_{2}$, with its $\mathrm{CB}$ below the $\mathrm{CB}$ of perovskite, selectively transfers electrons from the perovskite's $\mathrm{CB}$ to the FTO anode. Similarly, CuSCN and PEDOT:PSS with its VB above the $\mathrm{VB}$ of perovskite, selectively transfer holes through the carbon particles to the FTO cathode. Carbon powder is used to increase the overall performance of the devices, increasing $\mathrm{V}_{\mathrm{oc}}, \mathrm{I}_{\mathrm{sc}}$ and efficiency from the experimental results. PEDOT:PSS layer was made on another FTO before sandwiching them together to minimize the risk of degradation from the perovskite layer, especially under high humidity condition (50-60\%) in which the fabrication is done.

\subsection{Fabrication}

The devices were fabricated on a $2 \times 2 \mathrm{~cm}$ Fluorinedoped tin oxide (FTO) coated glass. The FTO glass was cleaned by sonication in water, acetone and ethanol for 5 minutes each. A mesoporous layer of $\mathrm{T}_{\mathrm{i}} \mathrm{O}_{2}$ was prepared by spin coating $\mathrm{T}_{\mathrm{i}} \mathrm{O}_{2}$ precursor solution $(0.2$ $\mathrm{M}$ titanium isopropoxide $+0.1 \mathrm{M} \mathrm{HCl}$ in anhydrous ethanol) at $1000 \mathrm{rpm}$ for $30 \mathrm{~s}$ and annealed at $425^{\circ} \mathrm{C}$ for 
30 minutes. The plate was then cooled to bring the temperature down to ambient. Perovskite precursor solution $(0.5 \mathrm{M}$ lead chloride $+1.5 \mathrm{M}$ methylammonium iodide in DMF) was deposited on top of the $\mathrm{T}_{\mathrm{i}} \mathrm{O}_{2}$ layer by spin coating it at $1000 \mathrm{rpm}$ for $30 \mathrm{~s}$ and then annealed at $120^{\circ} \mathrm{C}$ for 30 minutes. The cell without HTL would then be sprinkled with carbon powder and sandwiched with another FTO glass on top of it.

CuSCN layer was made by spin coating CuSCN solution (0.05 M CuSCN in dipropyl sulphide) at 500 rpm for $30 \mathrm{~s}$ and dried at $80^{\circ} \mathrm{C}$ for 20 minutes. Carbon powder is then sprinkled on top of the $\mathrm{CuSCN}$ layer. PEDOT:PSS layer was prepared in another FTO glass due to its tendencies to degrade the perovskite layer. The glass with PEDOT:PSS is then heated at $120^{\circ} \mathrm{C}$ until it is fully dry. Carbon powder was then sprinkled on the FTO glass with perovskite and the PEDOT:PSS coated FTO was placed on top of it. The fabrication process is pictured in Figure 3. The fabrication process is generally the same for the device without HTL and CuSCN, but PEDOT:PSS skips $5^{\text {th }}$ step because carbon was already sprinkled on top of them after the annealing of PEDOT:PSS.

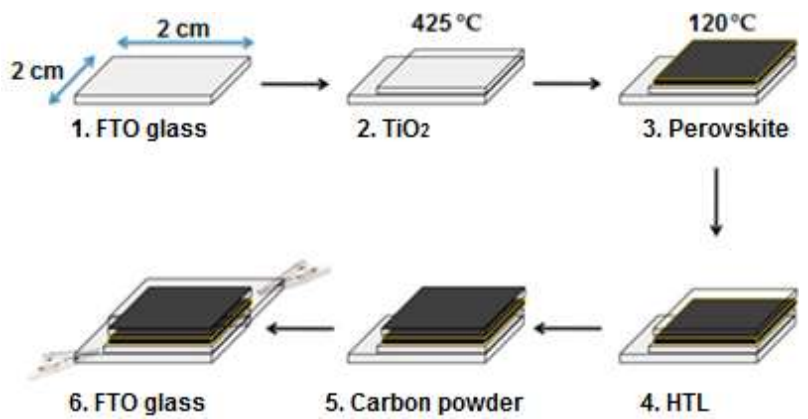

Fig. 3. Fabrication procedure

Table 1. Photovoltaic parameters

\begin{tabular}{|c|c|c|c|}
\hline Parameter & $\begin{array}{c}\text { Without } \\
\text { HTL }\end{array}$ & PEDOT:PSS & CuSCN \\
\hline $\mathrm{V}_{\mathrm{oc}}(\mathrm{V})$ & 0.12 & 0.22 & 0.24 \\
\hline $\mathrm{I}_{\mathrm{sc}}(\mathrm{mA})$ & 1.24 & 1.71 & 1.79 \\
\hline $\mathrm{FF}$ & 0.26 & 0.27 & 0.27 \\
\hline$\eta(\%)$ & 0.04 & 0.08 & 0.09 \\
\hline
\end{tabular}

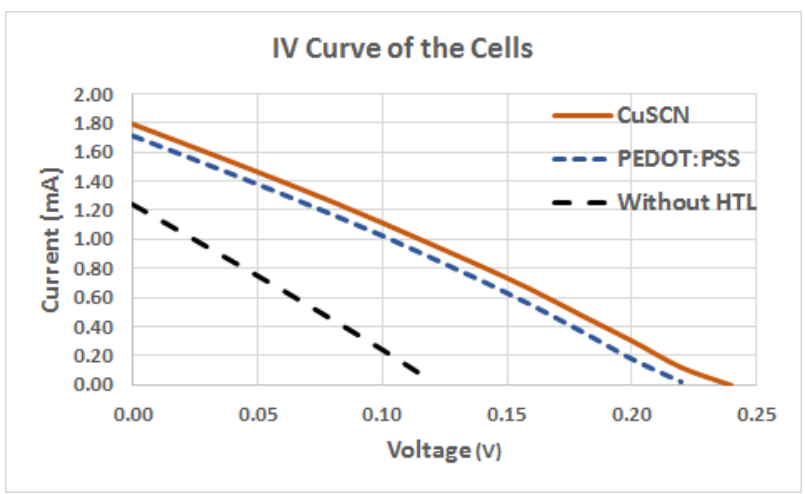

Fig. 4. IV curve of the fabricated solar cells

\section{Results and Discussion}

Dark IV curve of the cells are obtained from semiconductor parameter analyser (SPA) HP4145B and performance under illumination of light was measured using multimeter when the cells were illuminated directly from the sun. Photovoltaic parameters of the fabricated devices are noted at Table 1 and the I-V curve is pictured at Figure 4. The cell without HTL performs the worst, having the lowest $\mathrm{V}_{\mathrm{oc}}, \mathrm{J}_{\mathrm{sc}}, \mathrm{FF}$ and efficiency while the device with $\mathrm{CuSCN}$ as the HTL performs the best, with $\mathrm{V}_{\mathrm{oc}}$ of $0.24 \mathrm{~V}, \mathrm{I}_{\mathrm{sc}}$ of $1.79 \mathrm{~mA}$ on a $2 \times 2 \mathrm{~cm}$ substrate, $0.27 \mathrm{FF}$ and efficiency of $0.09 \%$.

The results on this paper are in line with the research conducted by Wolfgang Tress et al ${ }^{[11]}$. It was reported that the role of HTL is to decrease recombination at the electrode and increase absorption of mesoporous $\mathrm{TiO}_{2}$ layer. The decrease of recombination is evident from an increased open circuit voltage in the devices with HTL, making them perform better than the device without HTL. HTL can reduce the mismatch of the energy level between the perovskite and the electrode and acts as a bridge so holes and electrons can move to the electrodes without any significant losses. The presence of HTL will make the electrons and holes able to move freely to the electrodes and thus, having higher parameters overall.

Solar cell with PEDOT:PSS as HTL has lower $\mathrm{V}_{\mathrm{oc}}$, $\mathrm{I}_{\mathrm{sc}}$ and efficiency than $\mathrm{CuSCN}$, this might be due to the mismatch of energy level between PEDOT:PSS and perovskite is higher than the CuSCN. PEDOT:PSS's work function is around $-5.2 \mathrm{eV}$ to $-4.9 \mathrm{eV}$ depending on the content of PSS, $\mathrm{CuSCN}$ is $-5.3 \mathrm{eV}$ while the HOMO level of $\mathrm{CH}_{3} \mathrm{NH}_{3} \mathrm{PbI}_{3}$ is $-5.4 \mathrm{eV}$. CuSCN's work function is closer to the HOMO level of perovskite and it will have higher open circuit voltage than PEDOT:PSS. The device with CuSCN also has higher short circuit current, this might be due to the fabrication process of PEDOT:PSS that use different layers. The layer of PEDOT:PSS might not be properly joined electrically, increasing the recombination around the perovskite, lowering the amount of electrons and holes that can be transmitted to the electrodes and thus 
lowering the current. At the time of measurement, a part of the perovskite in the device with PEDOT:PSS has turned yellow, meaning some area of the perovskite has already degraded. More mismatch of energy level, PEDOT:PSS layer that might not properly be properly connected electrically and degradation of the perovskite in the PEDOT:PSS device might contribute to the lower performance of the device compared to the CuSCN.

The fabricated cells have relatively low $\mathrm{V}_{\mathrm{oc}}, \mathrm{I}_{\mathrm{sc}}, \mathrm{FF}$ and efficiency that can be attributed to the environment of fabrication. The cells were fabricated under high humidity (higher than 50\%) without clean room or glove box. Perovskite is really susceptible to moisture $^{[9]}$. Moisture and high humidity can degrade part the perovskite due to the decomposition series catalysed by water and thus, lowering their overall performance. Perovskite degradation is even more visible in PEDOT:PSS. If handled incorrectly or the layer isn't fully dry, PEDOT:PSS can degrade the perovskite which can be seen with naked eyes. The colour of the perovskite layer would turn yellow, signifying the loss of the depos within perovskite layer. The relatively poor FF might also be caused by the lack of metal contact. The mismatch of energy level from the HTL and the electrode might hinder the movement of holes and electrons, lowering the performance. Electrical conductivity of interfacial layers and metal contact is also an important factor determining device performance because charge transport occurs through the interfacial layers from perovskite absorbers and through the interfacial layer to the metal contact ${ }^{[12]}$. High electrical conductivity can increase the amount of charges that can be collected in the electrode with minimized recombination as long as charge balance between electrons and holes is stable in the solar cell device.

Higher open circuit voltage, short circuit current and FF can be achieved by using appropriate interfacial layers between the electrodes and perovskite layer. Perovskite should use a hole transport layer and electron transport layer with well-matching ionization energy and electron affinity to those of perovskites to facilitate higher charge transfer from perovskite to interfacial layers. The use of interfacial layers with high electrical conductivity is also favourable to make efficient charge transport and extraction to each electrode. Energy-level tailoring is one of the most effective ways to directly increase device performance. Appropriate energy-level tailoring can increase $\mathrm{V}_{\text {oc }}$ and facilitate charge transfer and extraction, which contribute to increasing $\mathrm{J}_{\mathrm{sc}}$ and $\mathrm{FF}$. In particular, $\mathrm{V}_{\mathrm{oc}}$ is determined by the quasi-Fermi level difference between hole transport layer and electron transport layer, reducing the energy offset between the valence band minimum of the perovskite and ionization energy of hole transport layer and between conduction band minimum of perovskite and electron affinity of electron transport layer is crucial to increase the quasi-Fermi level difference ${ }^{[12]}$. As such, using the matching materials is important to increase the overall performance of the fabricated solar cells.

\section{Conclusion}

In summary, we have demonstrated the effect of HTL to the performance of the solar cell. HTL can decrease the recombination around the electrode and increase the $\mathrm{V}_{\mathrm{oc}}, \mathrm{I}_{\mathrm{sc}}, \mathrm{FF}$ and efficiency of the cell. PEDOT:PSS performs worse than CuSCN due to having more mismatch of energy level, HTL layer that might not be properly connected electrically and degradation of the perovskite in the PEDOT:PSS device. The best performing device is perovskite solar cell with $\mathrm{CuSCN}$ as HTL layer, i.e. $\mathrm{V}_{\mathrm{oc}}$ of $0.24 \mathrm{~V}, \mathrm{I}_{\mathrm{sc}}$ of $1.79 \mathrm{~mA}, 0.27$ FF and efficiency of $0.09 \%$. The low FF in the devices fabricated in this paper might be due to the lack of metal contact and the relatively high humidity of the fabrication. Despite the current shortcomings, this paper identifies the roles of HTL in a perovskite solar cell and analysed the difference of performance between CuSCN and PEDOT:PSS.

This work has been supported by International publications indexed for the Final Project of Students (PITTA) Research Grant 2018 from Universitas Indonesia with $\mathrm{Nr}$. 2502/UN2.R3.1/HKP.05.00/2018

\section{References}

1. National Energy Council (DEN), Outlook Energy Indonesia 2014, Jakarta: Ministry of Energy and Mineral (2014)

2. Directorate General of Renewable Energy and Energy Conservation, Statistik EBTKE 2016, Jakarta: Ministry of Energy and Mineral (2016)

3. [Online].Available:https://www.nrel.gov/pv/assets/i mages/efficiency-chart.png

4. J. Burschka, et al., Sequential deposition as a route to high-performance perovskite-sensitized solar cells, Nature, 499, 316-319 (2013)

5. Q. Q. Lin, et al., Electro-optics of perovskite solar cells, Nat. Photonics, 9, 106-112 (2015)

6. C. Wehrenfennig, et al., Charge-carrier dynamics in vapour-deposited films of the oranolead halide perovskite CH3NH3PbI3-xClx, Energy Environ Sci, 7, 2269-2275 (2014)

7. G. Li, R. Zhu, Y. Yang, Polymer solar cells, Nat. Photonics, 6, 153 (2012)

8. T. Liu, K. Chen, Q. Hu, R. Zhu, Q. Gong, Inverted Perovskite Solar Cells: Progresses and Perspectives, Adv. Energy Mater., 6 Issue 17 (2016) 
9. Bárbara Andrade de Carvalho, et al., Highly Stable Perovskite Solar Cells Fabricated Under Humid Ambient Conditions, IEEE Journal of Photovoltaics, 7, no. 2, (2017)

10. J. Liu, et al., Novel Low Cost Hole Transporting Materials for Efficient Organik-inorganik Perovskite Solar Cells, PVSC 2015 IEEE 42 ${ }^{\text {nd }}$ (2015)

11. W. Tress et al., The role of the hole-transport layer in perovskite solar cells - reducing recombination and increasing absorption, PVSC 2014 IEEE $40^{\text {th }}(2014)$

12. H. Kim, K.-G. Lim and T.-W. Lee, Planar heterojunction organometal halide perovskite solar cells: roles of interfacial layers, Energy Environ. Sci., 9, 12-30 (2016)

13. S. Patwardhan et al., Introducing Perovskite Solar Cells to Undergraduates, J. Phys. Chem. Lett., 6(2), 251-255 (2015)

14. Q. A. Akkerman et al., Strongly emissive perovskite nanocrystal inks for high-voltage solar cells, Nat. Energy, 2 (2016)

15. H. Wang et al., The Effect of Carbon Black in Carbon Counter Electrode for $\mathrm{CH} 3 \mathrm{NH} 3 \mathrm{PbI} 3$ /TiO 2 heterojunction solar cells, RSC adv. (2015)

16. P. Qin et al., Inorganic hole conductor-based lead halide perovskite solar cells with $12.4 \%$ conversion efficiency, Nat. Comm, 5 (2014) 